\title{
Real-time view of AMP-mediated killing
}

Atomic force microscopy (AFM) has provided microbiologists with powerful images of bacterial cells and cell surface structures at the nanometre scale. However, image acquisition times of several minutes per frame have limited the use of AFM for studying highly dynamic cellular processes. Publishing in Nature Nanotechnology, Fantner et al. now describe the use of a new high-speed AFM approach to follow the kinetics of antimicrobial peptide (AMP) activity on a bacterial cell surface.

Damage caused to the bacterial cell surface by AMPs has been observed previously using electron microscopy and conventional AFM. However, only the end point of membrane disruption is visualized using such approaches. To investigate the kinetics of the early stages of AMP-mediated membrane disruption, the authors used a recently developed high-speed AFM approach in which micro-fabricated small cantilevers are used to scan the sample surface. The low mass of these small cantilevers (which is

AFM will enable the kinetics of events at the bacterial cell surface to be explored both in real time and at the nanometre scale.

\section{1,000-fold less than that of conven-} tional AFM cantilevers) allows an image acquisition speed of one frame every 13 seconds while keeping the force applied to the sample low, thereby preventing damage to the bacteria being imaged.

The authors observed that on addition of the AMP CM15 to Escherichia coli cells the normally smooth appearance of the cell surface changed, becoming corrugated. Although all cells in the field of view eventually became corrugated and died, the time of onset of this

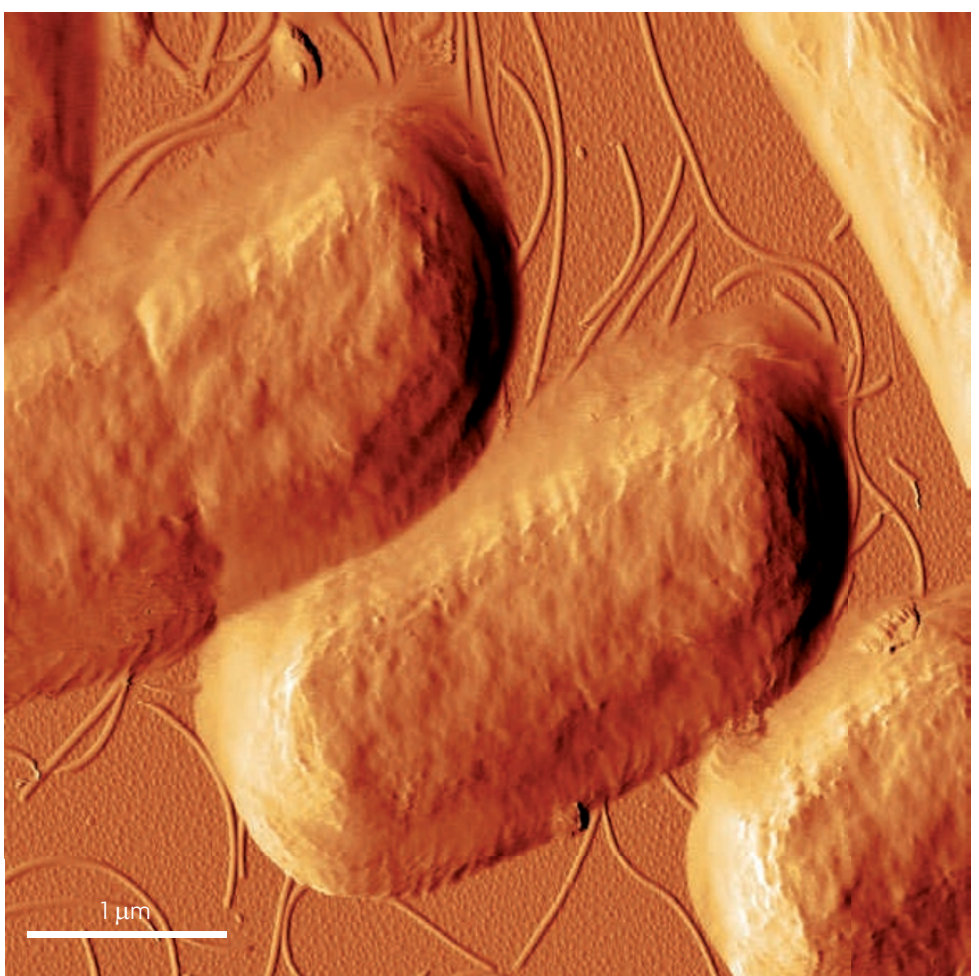

High-speed atomic force microscopy image of an Escherichia coli cell following incubation in the presence of the antimicrobial peptide CM15 for 5 minutes. The scale bar represents $1 \mu \mathrm{m}$. Image modified, with permission, from Fantner, G. E. et al. @ (2010) Macmillan Publishers Ltd. All rights reserved.

morphological change ranged from 40 seconds to 4 minutes for different cells. However, once membrane disruption had begun, the time for each bacterium to complete the morphological change was much more consistent, with over half of the bacteria becoming completely corrugated in 52 seconds (with a standard deviation of \pm 16 seconds). The authors propose that bacterial killing by CM15 proceeds in a two-step process consisting of an incubation phase of variable length followed by an execution phase, in which most of the damage to the bacteria occurs in less than a minute.

As these results show, high-speed AFM will enable the kinetics of events at the bacterial cell surface to be explored both in real time and at the nanometre scale.

Andrew Jermy

ORIGINAL RESEARCH PAPER Fantner, G. E.

et al. Kinetics of antimicrobial peptide activity measured on individual bacterial cells using highspeed atomic force microscopy. Nature Nanotech. 14 Mar 2010 (doi:10.1038/nnano.2010.29) 